\title{
Recent Trends in Conducting School-based Experimental Functional Analyses
}

\author{
Stacy L. Carter \\ Abstract \\ Demonstrations of school-based experimental functional analyses have received limited attention within the \\ literature. School settings present unique practical and ethical concerns related to the implementation of \\ experimental analyses which were originally developed within clinical settings. Recent examples have made definite \\ contributions toward addressing the problems related to incorporation of experimental functional analyses in school \\ settings. The advances made in these areas are reviewed and discussed in an effort to further the potential use of \\ these procedures with school settings. \\ Keywords: functional analysis, applied behavior analysis, school settings, problem behavior
}

Consultation in school settings can be a very complex process that requires an extensive knowledge of school-related problems, understanding of how interpersonal relationships are developed and maintained, and competencies in training specific skills to other practitioners. While evidence for the effectiveness of school-based consultation exists (Gutkin, \& Curtis, 1999; Kratochwill, Elliott, \& Busse, 1995; Sheridan, Welch, \& Orme, 1996), the incorporation of experimental functional analysis (EFA) within school-based consultation is still in need of further refinement. EFA involves observing, recording, and evaluating potential variables maintaining a specific target behavior during a process that involves systematically manipulating these potential maintaining variables (Iwata, Dorsey, Slifer, Bauman, \& Richman, 1982/1994; Vollmer, \& Northrup, 1996). One of the primary problems that exists with including experimentalfunctional analysis procedures within school settings is the issue of portability from clinic al to educational settings. These procedures were initially developed within clinical settings that allowed for a high degree of situational, methodological, and analytical control and rigor which may present difficulties when introducing these procedures into school settings. This paper provides a review of some of the current practices involving experimental functional analysis within school settings, a discussion of some of the related issues, and some recommendations for future research and practice.

\section{OVERVIEW}

While EFAs involve structured manipulations of environmental events (Cone, 1997), the term functional assessment or functional behavioral assessment is the frequently used umbrella term to describe all the procedures involved in assessing the function of a behavior including activities such as interviews, rating scales, and EFA. Ervin et al. (2001) reviewed the empirical literature on school-based functional assessment that appeared in published journals from 1980 to 1999. The review included 100 articles and revealed several limitations in the practice of school-based functional assessment. Among the limitations were a lack of consistent methodologies used, minimal examples of demonstrated use of the procedures across specific populations, behaviors, settings, etc., and few studies involving training of school personnel to conduct functional assessments. In addition, the review by Ervin et al. indicated that demonstrations of schoolbased experimental functional analyses appeared limited due to practical and ethical considerations. These concerns include the need for expert consultants to carry out the procedures, the need for highly controlled situations, the length of time needed to conduct analyses, and the potential risks associated with manipulating putative controlling variables.

Since the publication of review by Ervin et al. several examples of how to systematically conduct functional behavioral assessments have been developed and demonstrated to be effective (Ervin et al., 2000; Doggett, Edwards, Moore, Tingstrom, \& Wiczynski, 2001; McComas, Goddard, Hoch, 2002; 
Mueller, Edwards, \& Trahant, 2003; Umbreit, Ferro, Liaupsin, \& Lane, 2007). While these models provide a framework for determining when and how to implement various direct and indirect assessments, they do not provide a detailed systematic approach for conducting schoolbased EFAs in a manner that can easily implemented with a variety of students displaying a wide range of problematic behaviors. These models primarily focus upon more indirect methods of interviews, rating scales, and anecdotal data. A model for developing specific variations and modifications to EFAs needed for conducting these procedures within school settings does not appear to available within the literature. While this type of model may be difficult to develop due to the numerous variations and modifications which may be relevant, there does appear to be some clear elements which can be gleaned from the recent literature on school-based EFAs.

\section{REVIEW OF LITERATURE}

Definite progress has been made to increase the portability of experimental functional analysis procedures from clinical settings to school settings (Broussard, \& Northrup, 1995; Northrup et al., 1995). The usefulness of these examples of conducting experimental functional analysis in school settings has been evident in the outcomes produced, although issues related to time intensiveness, deviations from clinical protocols, involvement of paraprofessionals, etc. still exist. In order to adapt EFA procedures to classroom settings, several modifications and variations of clinical protocols have been employed.

Mueller, Sterling-Turner, \& Moore (2005) stated that further refinements of EFA procedures were needed to promote increased utilization within school settings. They pointed out that the procedures were initially developed within clinical settings and variables relevant to clinical participants were incorporated into the EFAs and those relevant variables within school settings might be quite different than variables important in clinical settings. Northrup et al. conducted an EFA within a classroom setting that involved two variations of an attention condition. Comparisons were made between teacher-delivered and peerdelivered attention for three participants who displayed typical classroom problem behaviors such as inappropriate vocalizations and out-of-seat behavior. Their results indicated that an EFA could be used to determine distinct forms of positive reinforcement such as peer-delivered attention which maintained the problem behaviors of all three participants. This method of limiting the number of conditions examined when conducting EFA in school settings appears to be one means of increasing the portability of these procedures from clinic to classroom settings.

Other studies have similarly limited the number of different conditions that were included in EFA's conducted within classrooms (Doggett et al., 2001; Mueller, Sterling-Turner, \& Scattone, 2001; Mueller et al., 2005). Mueller et al. (2005) described a classroom-based EFA condition to develop an intervention to decrease the tantrum behavior of a 6-year-old boy with autism. They used a hypothesisdriven approach based on prior descriptive data to evaluate attention and escape as maintaining variables within an EFA. The EFA conditions initially involved an attention condition which involved provision of attention upon the occurrence of tantrum behavior and an escape condition which involved removal of task demands upon occurrence of tantrum behavior. A follow-up condition was also developed which was described as escape-to-attention and involved removal of task demands upon occurrence of tantrum behavior and subsequent attention during the break period from the task. Their findings revealed that the escape-to-attention condition was necessary to appropriately determine the function of the behavior and develop an effective intervention. Their study demonstrated a potential limitation of EFAs conducted in school settings which do not typically incorporate specialized conditions. In addition, they also speculated that other specialized EFA conditions might also prove beneficial in school-based settings such as an escape-to-preferred activities condition.

While limiting the number of conditions incorporated into a school-based EFA could potentially restrain the findings, the addition of well designed follow-up conditions may be viable means of addressing this issue. Mueller, Wilczynski, Moore, Fusilier, \& Trahant (2001) conducted follow-up 
analysis to a schoolbased EFA which involved antecedent manipulation of highly preferred and less preferred items. The follow-up analysis was benefic ial toward developing an intervention to reduce the aggression of an 8-year-old boy with autism. They determined that the follow-up analysis was necessary in addition to the initial EFA to recommend an effective intervention. Similarly, Moore, Mue ller, Dubard, Roberts, and Sterling-Turner (2002) conducted a follow-up analysis to clarify confounding data collected during the tangible condition of a school-based EFA involving a 6-year-old girl who engaged in selfinjurious behavior. Their follow-up analysis involved comparing tangibles conditions that did and did not include verbal attention. They determined that verbal attention within a tangible condition introduced confound to the interpretation of the initial EFA and the follow-up was necessary to adequately interpret the data. Similarly, Carter, Devlin, Doggett, Harber, and Barr (2004) found a follow-up analysis was necessary to clarify the confound produced by the inclusion of tangible items within an alone condition of a school-based EFA. Based upon these studies, the usefulness of follow-up analysis may be an important component toward developing a comprehensive model for conducting school-based EFAs. While followup analysis may be valuable, they should be considered along with overall attempts to minimize the time intensiveness of conducting schoolbased EFAs.

Mueller, Sterling-Turner, et al. (2001) described using a brief EFA based upon prior descriptive assessment data to assess the hand flapping behavior of a 5-year-old boy in a general classroom setting. They recruited teacher assistance during the EFA which compared a low frequency task demand condition with a high frequency demand condition. Their hypothesis-driven approach demonstrated that descriptive data can inform the development of EFA conditions to test possible functions of target behavior while limiting the number of experimental conditions and making the EFA more efficient and practical for implementation within a classroom setting. Their study was an example of recommendations on the future of EFA's made by Carr (1994), who stated that comprehensive descriptive assessments should be a method used in order to individualize EFA conditions and increase the potential relevance of the analysis outcomes. This recommendation by Carr appears to be highly applicable to EFA's conducted within school settings due to the limited allowances from school administrators, lack of personnel adequately trained to complete EFA's, and environmental limitations such as the presence of other students and classroom layouts.

As noted by Repp (1994), the introduction of EFA into classroom settings was initially difficult due to reluctance on the part of administrators to allow these types of procedures to be conducted due to the potential for high rates of problem to occur during the conditions. Moore et al. (2002) reported omitting the alone condition of an EFA at the request of school personnel. Repp also explained that although these administrators were resistant to frequent problem behaviors during EFA conditions, they appeared to be accepting of high rates of problem behaviors in typical classroom settings, although they wanted these behaviors to decrease.

One possible means of addressing this concern in addition to limiting the number of sessions that are included in an EFA could be to train educational personnel to conduct EFAs with less reliance on outside consultants. Having competently trained educational personnel within the school system might promote the use of these procedures within schools. This might also increase the portability of these procedures from clinical practices by integrating techniques developed by educational personnel who are more directly involved with the students being assessed than would be typical of an outside consultant.

Scott, McIntyre, Liaupsin, Nelson, \& Conroy (2004) evaluated differences in hypotheses derived from functional behavioral assessments by school-based teams and those hypotheses developed by experts. They found poor agreement between the hypotheses developed by the teams and those developed by the experts. The differences in the hypotheses that were generated were dependent upon the amount and type of information that was provided by the assessments with more information from the functional behavior assessments leading to more disagreement. They concluded that hypotheses derived by experts 
were limited by their lack of contact with the students and the hypotheses developed by school based teams were limited by their lack of expertise with the procedures. The Scott et al. study may offer some insight into how the portability of EFA procedures into school settings could be improved by incorporating information and techniques which may be exclusive only to school personnel. In other words, school personnel may be privy to certain information that an outside consultant may not readily procure within the limited amount of contact that they have with a student. This type of information could be useful toward the development of specific EFA conditions and reduce some of the limitations associated with assessments conducted within analog versus natural environments such as lack of incorporation of necessary variables from the natural environment and lack of generalization of findings. In addition, information from school personnel may readily inform the scheduling and arrangement of the environment within the school as well as determining the availability of other services such as the presence of a school nurse to assist in case of injury especially for EFA of dangerous behaviors such as self-injury.

It appears that many of the EFAs described in the recent literature have been conducted within vacant classrooms which highly resemble clinical settings (St. Peter et al., 2005; Moore et al., 2002; Mueller, Wiczynski, et al., 2001). While this may be a requirement in many cases, it is possible that school personnel could suggest other alternatives that would allow for EFAs to be conducted in more natural classroom settings with other students present while still maintaining an adequate level of experimental control over the conditions such as the examples provided by Mueller, Edwards, \& Trahant (2003), Mueller, Sterling-Turner, et al (2001), and Mueller, Sterling-Turner, et al. (2005). In order for these types of developments to take place, school personnel need to become more competent EFA procedures. There appears to be several EFA examples which incorporate school personnel (Erbas, TekinIftar, \& Yucesoy, 2006; Erbas, Yucesoy, Turan, Ostrosky, 2006; Kamps, Wendland, \& Culpepper, 2006), but these examples indicate that school personnel continue to need a great deal of support from an expert consultant.

Another variation of the more commonly used EFA is a structural analysis. Stichter \& Conroy (2005) described structural analyses as procedurally similar to EFA, but with a focus on discerning relationships between antecedent variables and target behaviors. While this methodology does not emphasize determination of variables maintaining target behaviors, it does allow for close examination of variables which set the occasion for target behaviors to occur. While the structural analysis methodology does not allow for the development of function-based interventions, it does have potential for offering a means for increasing the portability and utilization of systematic assessments of contextual variables within a classroom setting.

The rationale for increased portability with these procedures relies on involving techniques that are already frequently used by teachers to problem solve academic and behavioral problems in classrooms. Teachers may frequently modify their instructional practices in attempts to find a "best fit" approach for teaching a student. Teachers may informally try techniques that include presenting instructional materials in different formats and venues such as orally, written, individually, in small groups, in large groups, etc. In addition, teachers may consider instructional variations that include more or less frequent prompts, student choices in instructionalpresentation, and arrangement of more or less demanding activities. Since teachers frequently engage in this type of informal problem solving, the structural analysis methodology which involves formalizing some of the informal techniques currently used by teachers would seem to increase the portability of these procedures from clinical settings to classroom settings and from clinically trained personnel to educators.

Several studies have demonstrated the usefulness of structural analysis procedures toward development of effective interventions in classroom settings (Hagan-Burke, Nurke, \& Sugai, 2007; Stichter, Lewis, Johnson, \& Trussell, 2004; Stichter, Sasso, \& Jolivette, 2004; Wheeler, Carter, Mayton, \& Thomas, 2002). Conroy \& Stichter (2003) compared a model for conducting a structural analysis 
involving analog probes with a correlational analysis model which involved repeated naturalistic observations and indicated that the structural analysis model required le ss complex data collection procedures, was more time efficient, and may improve implementation of interventions due to teacher involvement in the assessment. Although more research is needed to examine the potential of using structural analyses in classroom settings, there appears to be potential for these procedures to be in place of an EFA in certain circumstances, to be used as complements to EFAs, and to enhance teacher implementation interventions.

\section{SUMMARY AND CONCLUSIONS}

In summary, the current trends for implementing EFAs in classroom settings appear to focus on developing methods that are structured, simplistic, time efficient, and easily incorporated by individuals who may lack previous training in conducting EFAs. The study by Mueller et al. (2005) incorporated the use of a paraprofessional to deliver contingencies within the EFA while a consultant collected data. In addition they relied upon prior descriptive data to minimize the number of condition considered relevant and conducted the EFA conditions during times considered most problematic for the student. Each of these factors appears to be highly relevant toward the future development of schoolbased EFAs especially when appropriate follow-up strategies can be developed to ensure accuracy in interpretation of findings (Moore et al, 2002; Mueller et al., 2001; Carter et al., 2004). Strategies to simplify the procedures involved in conducting a schoolbased EFA appear to be highly appealing and important to the future utilization of these procedures in school setting. Hypothesis-driven approaches which may involve relying on prior descriptive data to include conditions considered highly relevant and exclude conditions that do not appear relevant have been demonstrated as an effective means of simplifying school-based EFAs (Mueller et al. 2001; Mueller et al., 2005; Northrup et al., 1995).

Strategies for making school based EFAs more time efficient are also related to using a hypothesis-driven approach to limit the number of conditions included in the EFA as well as decreasing the number of sessions conducted and the length of session time. These recent developments address some of the limitations described by Ervin et al. but further progress is needed to promote more portability of these procedures from clinical to educational settings. In addition, the need to develop specialized conditions for school-based EFAs may also be an important future direction for research in order to further remove these procedures from a clinical model and make these procedures uniquely designed to function within a school setting. Taking procedures initially developed within a clinical setting and utilizing them in a school setting without modification would most likely result in several apparent difficulties. By modifying these clinically developed procedures into a formalized school-based model while maintaining an appropriate level of scientific rigor would appear to be the most likely method for ensuring the continued inclusion and usefulness of these procedures in school settings.

\section{References}

Broussard, C. D., \& Northrup, J. N. (1995). An approach to functional assessment and analysis of disruptive behavior in regular education classrooms. School Psychology Quarterly, 10, 151-164.

Carr, E. G. (1994). Emerging themes in the functional analysis of problem behavior. Journal of Applied Behavior Analysis, 27, 393-399.

Carter, S. L., Devlin, S., Doggett, R. A., Harber, M. M., \& Barr, C. (2004). Determining the influence of tangibles on screaming and handmouthing following an inconclusive functional analysis. Behavioral Interventions, 19, 51-58. 
Cone, J. D. (1997). Invited essay. Issues in functional analysis in behavioral assessment. Behaviour Research and Therapy, 35, 259-275.

Conroy, M. A., \& Stichter, J. P. (2003). The application of antecedents in the functional assessment process: Existing research, issues, and recommendations. The Journal of Special Education, 37, $15-25$.

Doggett, R. A., Edwards, R., Moore, J., Tingstrom, D., \& Wilczynski, S. (2001). An approach to functional assessment in general education classroom settings. School Psychology Review, 30, 313-328.

Erbas, D., Tekin-Iftar, E. \& Yucesoy, S. (2006). Teaching special education teachers how to conduct functional analysis in natural settings. Education and Training in Developmental Disabilities, 41, 28-36.

Erbas, D., Yucesoy, S., Turan, Y., \& Ostrosky, M. M. (2006). Turkish special education teachers' implementation of functional analysis in classroom settings. Education and Training in Developmental Disabilities, 41, 155-162.

Ervin, R. A., Radford, P. M., Bertsch, K., Piper, A. L., Ehrhardt, K. E., \& Poling, A. (2001). A descriptive analysis and critique of the empirical literature on school-based functional assessment. School Psychology Review, 30, 193-210.

Gutkin, T. B., \& Curtis, M. J. (1999). School-based consultation theory and practice: The art and science of indirect service delivery. In C. R. Reynolds \& T. B. Gutkin (Eds.), The handbook of school psychology ( $3^{\text {rd }}$ ed. pp. $\left.598-637\right)$. New York: Wiley.

Hagan-Burke, S., Burke, M. D., \& Sugai, G. (2007). Using structural analysis and academic-based intervention for a student at risk of EBD. Behavioral Disorders, 32, 175-191.

Iwata, B. A., Dorsey, M. F., Slifer, K. J., Bauman, K. E., \& Richman, G. S. (1994). Toward a functional analysis of self-injury. Journal of Applied Behavior Analysis, 27, 197-209. (Reprinted from Analysis and Intervention in Developmental Disabilities, 2, 3-20, 1982).

Kamps, D., Wendland, M., \& Culpepper, M. (2006). Active teacher participation in functional behavior assessment for students with emotional and behavioral disorders risks in general education classrooms. Behavioral Disorders, 31, 128-146.

Kratochwill, T. R., Elliott, S. N., \& Busse, R. T. (1995). Behavior consultation: A five-year evaluation of consultation and client outcomes. School Psychology Quarterly, 10, 87-117.

McComas, J., Goddard, C., \& Hoch, H. (2002). The effects of preferred activities during academic work breaks and task engagement and negatively reinforced destructive behavior. Education and Treatment of Children, 25, 103-112.

Mueller, M. M., Edwards, R. P., \& Trahant, D. (2003). Translating multiple assessment techniques into an intervention selection model for classrooms. Journal of Applied Behavior Analysis, 36, 563573. 
Mueller, M. M., Sterling-Turner, H. E., \& Moore, J. W. (2005). Towards developing a classroom-based functional analysis condition to assess escape-to-attention as a variable maintaining problem behavior. School Psychology Review, 34, 425-431.

Mueller, M. M., Sterling-Turner, H. E., \& Scattone, D. (2001). Functional assessment of hand flapping in a general education classroom. Journal of Applied Behavior Analysis, 34, 233-236.

Mueller, M. M., Wilczynski, S. M., Moore, J. W., Fusilier, I., \& Trahant, D. (2001). Antecedent manipulations in a tangible condition: The effects of stimulus preference on aggression. Journal of Applied Behavior Analysis, 34, 237-240.

Moore, J. W., Mueller, M. M., Dubard, M., Roberts, D. S., \& Sterling-Turner, H. E. (2002). The influence of therapist attention on self-injury during a tangible condition. Journal of Applied Behavior Analysis, 35, 283-286.

Northrup, J., Broussard, C., Jones, K., George, T., Vollmer, T. R., \& Herring, M. (1995). The differential effects of teacher and peer attention on the disruptive classroom behavior of three children with a diagnosis of attention deficit hyperactivity disorder. Journal of Applied Behavior Analysis, 28, 227-228.

Sheridan, S. M., Welch, M., \& Orme, S. F. (1996). Is consultation effective? A review of outcome research. Remedial and Special Education, 17, 341-354.

Stichter, J. \& Conroy, M. (2005). Using structural analysis in natural settings: A responsive functional assessment strategy. Journal of Behavioral Education, 14, 19-34.

Stichter, J., Lewis, T., Johnson, N., \& Trussell, R. (2004). Toward a structural assessment: Analyzing the merits of an assessment tool for a student with EBD. Assessment for Effective Intervention, 30, $25-40$.

Stichter, J. P., Sasso, G. A., Jolivette, K. (2004). Structural analysis and intervention in a school setting: Effects on problem behavior for a student with an emotional/behavioral disorder. Journal of Positive Behavior Intervention, 6, 166-177.

St. Peter, C. C., Vollmer, T. R., Bourret, J. C., Borrero, C. S. W., Sloman, K. N., Rapp, J. T. (2005). On the role of attention in naturally occurring matching relations. Journal of Applied Behavior Analysis, 38, 429-444.

Umbreit, J., Ferro, J.. Liaupsin, C., \& Lane, K. (2007). Functional behavioral assessment and functionbased intervention: An effective practical approach. Upper Saddle River, NJ: Prentice-Hall.

Wheeler, J.J., Carter, S.L., Mayton, M.R., \& Thomas, R.A. (2002). Structural analysis of instructional variables and their effects on task engagement and self-aggression. Education and Training in Mental Retardation and Developmental Disabilities, 37, 391-398.

\section{Author contact information:}

Stacy L. Carter

Department of Educational Psychology and Leadership

Texas Tech University;

P.O. BOX 41071

Lubbock, TX 79409-1071

stacy.carter@ ttu.edu. 